\title{
Enzimas miocárdicas na cirurgia de revascularização sem circulação extracorpórea
}

\author{
Paulo Roberto SOLTOSKI ${ }^{*} * *, * * *$, Giuseppe D'ANCONA**, Carlos Alberto Mussel BARROZO*, \\ Fernando Mendes SANT'ANNA*, Anderson Wilnes PEREIRA* , Jacob BERGSLAND**, \\ Tomas Antônio SALERNO ${ }^{* *} * * *$, Anthony L. PANOS ${ }^{* * *}$
}

RBCCV 44205-492

\begin{abstract}
Soltoski P R, D'Ancona G, Barrozo C A M, Sant'Anna F M, Pereira A W, Bergsland J, Salerno T A, Panos $A L$ - Enzimas miocárdicas na cirurgia de revascularização sem circulação extracorpórea. Rev Bras Cir Cardiovasc 2000; 15(2): 105-8.
\end{abstract}

RESUMO: Introdução: A cirurgia cardíaca com o coração batendo está sendo cada vez mais utilizada para minimizar danos cerebrais, renais e outros. Porém, a incidência de infarto nos períodos per e pós-operatório e seu efeito a longo prazo, especialmente quando esta técnica é comparada às tradicionais, ainda não foi totalmente esclarecida.

Casuística e Métodos: Um estudo retrospectivo de 303 pacientes (122 sem CEC, 181 com CEC) foi realizado de fevereiro/97 a fevereiro/99. Dosagens de CK-MB e eletrocardiogramas foram avaliados nos períodos per e pós-operatório, troponina $\mathrm{T}(\mathrm{TnT})$ foi medida no período pósoperatório. Os dois grupos foram comparáveis quanto a idade (65 \pm 10 anos vs. $65 \pm 9$ anos), classificações CCS e NYHA.

Resultados: O número médio de enxertos com CEC foi de 3,10 vs. 2,26 sem CE. Infarto perioperatório, assim como morbidade e mortalidade (7/181 vs. 6/122) foram comparáveis. Apesar de mais elevados no grupo com CEC, os níveis pós-operatórios de CK-MB e troponina T não atingiram diferença estatística, tendo sido observados 21 infartos agudos por critérios bioquímicos que se distribuíram igualmente entre os grupos. Uma correlação inversa entre o número de enxertos e a liberação de troponina foi observada na fase inicial da nossa experiência sem CEC. Não houve relação entre a localização do enxerto e a incidência de infarto ou liberação de troponina.

Conclusão: A proteção miocárdica, morbidade e mortalidade obtidas com as duas técnicas de revascularização estudadas foram semelhantes. Considerando os efeitos adversos da CEC, já documentados na literatura, particularmente sobre os sistemas neurológico, respiratório e renal sugerimos esta técnica como uma alternativa segura para a revascularização miocárdica.

DESCRITORES: Miocárdio, enzimologia. Revascularização miocárdica, métodos. Revascularização miocárdica, efeitos adversos. Revascularização miocárdica, mortalidade. Circulação extracorpórea, efeitos adversos. Circulação extracorpórea, mortalidade.

Trabalho realizado em: Clinica Santa Helena, Cabo Frio, RJ, Brasil; State University of New York at Buffalo, Kaleida Health System, Buffalo, NY, USA; Buffalo VA Medical Center, Buffalo, NY, USA.

Apresentado ao $27^{\circ}$ Congresso Nacional de Cirurgia Cardíaca. Rio de Janeiro, RJ, 23 a 25 de março, 2000.

${ }^{*}$ Da Clinica Santa Helena.

${ }^{* *}$ Da University of New York at Buffalo, Kaleida Health System.

*** Do Buffalo VA Medical Center.

Endereço para correspondência: Paulo Roberto Soltoski. Rua Comendador Araújo, 323 - $12^{\circ}$ andar - Cj. 127 - Curitiba - PR - Brasil. CEP $80420-903$.

Tel: (041) 9977-0169/322-8890. e-mail: paulosoltoski@uol.com.br 
Soltoski P R, D’Ancona G, Barrozo C A M, Sant'Anna F M, Pereira A W, Bergsland J, Salerno T A, Panos A L - Enzimas miocárdicas na cirurgia de revascularização sem circulação extracorpórea. Rev Bras Cir Cardiovasc 2000; 15(2):105-8.

\section{INTRODUÇÃO}

As novas técnicas para revascularização miocárdica completa sem circulação extracorpórea (CEC), frutos da criação de estabilizadores capazes de imitar as técnicas já consagradas pelo tempo, estão hoje sendo postas à prova por motivos diferentes daqueles considerados há poucos anos.

Atualmente, questões relacionadas a dificuldades técnicas de construção de anastomoses passaram para segundo plano e a dúvida principal tornou-se a qualidade da proteção miocárdica obtida através da revascularização sem CEC. Esta passagem do nível técnico para a análise dos distúrbios metabólicos, característica da medicina em geral freqüentemente observada na operação, busca questionar a necessidade da criação de uma nova modalidade de tratamento para a doença que possui o mais bem documentado tratamento cirúrgico da história da medicina, a cirurgia de revascularização miocárdica. Vários trabalhos vêm demonstrando a diminuição da morbidade na revascularização sem CEC, especialmente nos casos onde o paciente possui função pulmonar, renal ou vascular cerebral limítrofe $(1,2)$.

Nosso trabalho foi dedicado a avaliar se a busca da provável proteção superior observada para vários órgãos com os acima citados não se daria em detrimento da qualidade da proteção miocárdica durante a operação sem CEC.

\section{CASUÍSTICA E MÉTODOS}

Os prontuários de 303 pacientes operados entre fevereiro de 97 e fevereiro de 99 foram analisados. Cento e oitenta e um pacientes foram revascularizados com CEC e 122 pacientes sem CEC. A avaliação pré-operatória, a técnica anestésica básica, equipe cirúrgica e os cuidados pós-operatórios foram os mesmos para os dois grupos.

Para a técnica sem CEC, todos os casos foram operados através de esternotomia mediana. O estabilizador utilizado foi o CTS multivessel stabilizer (Cupertino, CA).

Após esternotomia e obtenção dos enxertos, o coração e a aorta ascendente foram inspecionados, o saco pericárdico foi suturado aos bordos da ferida cirúrgica e um ponto de vicryl no 1 (Ethicon, Sommerville, NJ) passado na altura do seio transverso do pericárdio posterior, muito próximo ao seio coronário (uma modificação da técnica apresentada por Lima e colaboradores) (3).

A coronária a ser anastomosada foi então preparada com a passagem de um garrote proximal almofadado de prolene 4-0 (Ethicon, Sommerville, $\mathrm{NJ})$ e a artéria alvo garroteada por cerca de cinco minutos para um período de pré-condicionamento isquêmico $(4,5)$. Um shunt intracoronário foi empregado sempre que possível (Anastaflo - Research Medical, Midvale, UT) e as anastomoses distais foram construídas com fio prolene 7-0 (Ethicon, Sommerville, NJ). Um soprador para visualização da anastomose foi utilizado em todos os casos (Visuflo - Research Medical, Midvale, UT). A exposição dos vasos foi obtida com uma combinação de tração e posicionamento do ponto de pericárdio posterior, associada a manobras com a mesa cirúrgica. Apenas após ótima exposição da artéria alvo, empregamos o estabilizador na região a ser anastomosada. As anastomoses proximais foram construídas com pinçamento parcial da aorta.

$\mathrm{Na}$ revascularização com CEC, em 181 pacientes foi usado oxigenador tipo membrana e cardioplegia sangüínea tépida hipercalêmica na proporção de $4: 1$, com indução anterógrada na maioria dos casos, seguida de manutenção contínua via retrógrada. Técnica cirúrgica padrão foi empregada para revascularização coronariana. Níveis séricos de CK-MB e TnT foram obtidos no período pósoperatório imediato, e em alguns casos 24 horas após a operação. Dosagens de CK-MB também foram obtidas no período pré-operatório em mais de $90 \%$ dos pacientes. Todos os estudos bioquímicos foram executados no laboratório da Instituição, utilizando-se para a dosagem de TnT reagentes de segunda geração (Boehringer Mannheim - GmbH, Mannheim, Germany), altamente específicos para o músculo cardíaco. Eletrocardiogramas pré e pósoperatório foram avaliados em $97 \%$ dos pacientes, sendo todos os laudos gerados ou revisados por cardiologistas. As complicações foram identificadas por uma auditora independente, responsável pela coleta de dados e preenchimento da ficha individual que é submetida a central de coleta de dados do New York State Database.

A análise estatística foi feita com o programa Statistica versão 5.1 (Statsoft, Tulsa, $\mathrm{OH}$ ). Análise de variância foi utilizada para testar variáveis tempo-dependentes. Quando F mostrou significância, confirmou-se com o teste de Scheffe post hoc. Correlação de Pearson foi utilizada para avaliação de dados com múltiplas variáveis. Todos os resultados foram apresentados como médias com $95 \%$ de intervalo de confiança. Significância foi assumida quando o valor de "p" mostrou-se menor do que 0,05.

\section{RESULTADOS}

Os grupos com CEC e sem CEC foram similares quanto à idade (65 \pm 10 anos vs. $65 \pm 9$ anos), 
Soltoski P R, D’Ancona G, Barrozo C A M, Sant'Anna F M, Pereira A W, Bergsland J, Salerno T A, Panos A L - Enzimas miocárdicas na cirurgia de revascularização sem circulação extracorpórea. Rev Bras Cir Cardiovasc 2000; 15(2):105-8.

classe funcional (NYHA) e classificação da angina pela CCS. O número médio de enxertos para a operação com CEC foi de 3,1 vs 2,26 sem CEC. Houve maiores níveis de CK-MB no pós-operatório com CEC (548 \pm 420 vs. $236 \pm 365)$, porém estes valores não atingiram significância estatística. Os valores pós-operatórios de TnT $(3,5 \pm 16$ com CEC vs. $3,5 \pm 17$ sem $C E C$ ) também não atingiram significância, mas observou-se uma correlação inversa entre o número de enxertos e a liberação de TnT no grupo sem CEC, particularmente na fase inicial da nossa experiência com esta técnica. Quando o número e localização dos enxertos foram comparados, observou-se que não havia correlação entre a localização das anastomoses distais e a incidência de infartos ou liberação de TnT. Vinte e um pacientes sofreram infarto agudo do miocárdio no período per-operatório (12 com CEC, 9 sem CEC), dos quais 17 apresentaram alterações eletrocardiográficas típicas. Os níveis médios de TnT nos pacientes infartados com CEC foi de 7,14 (4 à 14), enquanto que os níveis sem CEC foram 7,3 (4 à 21). A mortalidade (7/ 181 vs. 6/122) foi comparável nos dois grupos.

\section{COMENTÁRIOS}

A cirurgia de revascularização sem CEC tornou-se um importante objeto de investigação nos últimos anos. Apesar de ainda não existirem grandes estudos randomizados, provavelmente porque esta técnica ainda vem sofrendo modificações muito freqüentes, existem muitos relatos de procedimentos comparando diferentes métodos de revascularização. KREJKA et al. (6) demonstraram que alterações perceptíveis de TnT são observadas tão cedo quanto uma hora após um insulto isquêmico miocárdico. Devido às suas características estruturais, a TnT, ao contrário da CK-MB ou mioglobina, não é afetada por manobras de ressuscitação ou desfibrilação (7) e não sofre reação cruzada com outras enzimas, sendo, portanto, altamente específica para a lise de células miocárdicas (8).
MAIR et al. (8) sugeriram que níveis de TnT superiores a $3,5 \mu \mathrm{g} / \mathrm{L}$ constituiriam infarto agudo do miocárdio em pacientes operados com cardioplegia cristalóide. Em nossa série, observamos 21 pacientes com estes níveis, estando ambos os grupos divididos em partes praticamente iguais (12 com CEC vs. 9 sem CEC). Dentre os pacientes que apresentaram critério enzimático para IAM, um número menor apresentou quadro clínico complicado, que evoluiu à morte. Quando comparamos a cirurgia sem CEC com a técnica de cardioplegia sangüínea tépida, que pode ser considerada um dos métodos mais eficazes de proteção miocárdica atual (9) não observamos diferenças estatisticamente significantes. Em contrapartida, BOUCHARD \& CARTIER (10), utilizando a CK-MB, demonstraram diminuição importante na incidência de IAM em casos de revascularização completa sem CEC.

\section{CONCLUSÃO}

O desenvolvimento tecnológico observado nos últimos anos possibilitou a criação de novas técnicas de revascularização miocárdica. Tais avanços sempre geraram ansiedade, particularmente quando o tratamento tradicional é considerado seguro e eficaz. Baseados nos dados obtidos, acreditamos que a cirurgia de revascularização sem CEC, no que diz respeito à proteção miocárdica, seja tão segura quanto a revascularização tradicional, pois marcadores tão eficientes quanto a troponina T e a CK-MB não demonstraram diferença entre os grupos. Portanto, sugerimos que ela seja parte integrante das opções de tratamento do doente coronariano, particularmente nos pacientes que ofereçam riscos maiores à CEC, como os portadores de doença pulmonar obstrutiva crônica, doença obstrutiva vascular cerebral ou que apresentem função renal comprometida. 
Soltoski P R, D'Ancona G, Barrozo C A M, Sant'Anna F M, Pereira A W, Bergsland J, Salerno T A, Panos A L-Enzimas miocárdicas na cirurgia de revascularização sem circulação extracorpórea. Rev Bras Cir Cardiovasc 2000; 15(2):105-8.

RBCCV 44205-492

Soltoski P R, D'Ancona G, Barrozo C A M, Sant'Anna F M, Pereira A W, Bergsland J, Salerno T A, Panos A L - Myocardial enzymes in off-pump CABG surgery. Rev Bras Cir Cardiovasc 2000; 15 (2):105-8.

ABSTRACT: Background: CABG surgery on the beating heart (off-pump) is increasingly used to lessen injury to the brain, kidneys and other organs. However, the perioperative incidence of myocardial injury and its effect on outcome vs conventional CABG (on-pump) remains unclear.

Material and Methods: Retrospective study of 303 patients (122 off-pump, 181 on-pump) after isolated CABG from Feb/97-Feb/99. CPK and EKG were obtained pre and post-op, MB fraction and troponin $\mathrm{T}$ levels were measured postoperatively. Complications were also recorded. The groups were comparable in terms of age ( $65 \pm 10$ vs $65 \pm 9 \mathrm{yr}$ ), CCS and NYHA class.

Results: Mean number of grafts was 3.10 on-pump vs 2.26 off-pump. Perioperative myocardial infarction, morbidity and mortality ( $7 / 181$ vs $6 / 122)$ were also comparable. There were higher postoperative CK levels in the on-pump group compared to the off-pump (548 \pm 420 vs $236 \pm 365)$. MB fraction was slightly higher in the on-pump group, but not significantly different (62 \pm 197 vs $29 \pm 46$ ) nor was troponin T levels ( $3.5 \pm 16$ vs $3.5 \pm 17$ ) were An inverse correlation between the number of grafts and troponin $T$ release in the off-pump group occurred early during our off-pump experience. There was no correlation between graft location and the incidence of infarcts or troponin $T$ release.

Conclusion: The higher troponin T release during our initial experience subsequently decreased. This suggests that improvements in operative technique (e.g. better exposure allowing more posterior grafts without surface trauma) may be responsible. Current techniques make off-pump CABG a safe alternative for revascularization.

DESCRIPTORS: Myocardium, enzimology. Myocardial revascularization, methods. Myocardial revascularization, adverse effects. Myocardial revascularization, mortality. Extracorporeal circulation, adverse effects. Extracorporeal circulation, mortality.

\section{REFERÊNCIAS BIBLIOGRÁFICAS}

1 Bergsland J, Hasnan S, Lewin A N, Bhayana J, Lajos T Z, Salerno T A - Coronary artery bypass grafting without cardiopulmonary bypass: an attractive alternative in high risk patients. Eur $J$ Cardiothorac Surg 1997; 11: 876-80.

2 Taylor K M - Central nervous system effects of cardiopulmonary bypass. Ann Thorac Surg 1998; 66(5 Suppl): S20-8.

3 Bergsland J, Karamanoukian $\mathrm{H}$ L, Soltoski $\mathrm{P}$ R, Salerno T A - "Single suture" for circumflex exposure in off-pump coronary artery bypass grafting. Ann Thorac Surg 1999; 68:1428-30.

4 Szmagala P, Morawski W, Krejca M, Gburek T, Bochenek A - Evaluation of perioperative myocardial tissue damage in ischemically preconditioned human heart during aorto coronary bypass surgery. J Cardiovasc Surg (Torino) 1998; 39: 791-5.

$5 \quad$ Murry C E, Jennings R B, Reimer K A - Preconditioning with ischemia: a delay of lethal cell injury in ischemic myocardium. Circulation 1986; 74: 1124-36.

6 Krejca M, Skiba J, Szmagala P, Gburek T, Bochenek
A - Cardiac troponin $T$ release during coronary surgery using intermittent cross-clamp with fibrillation, on-pump and off-pump beating heart. Eur $J$ Cardiothorac Surg 1999; 16: 337-41.

7 Mullner $\mathrm{M}$, Oschatz $\mathrm{E}$, Sterz $\mathrm{F}$ et al. - The influence of chest compressions and external defibrillation on the release of creatine kinase-MB and cardiac troponin $T$ in patients resuscitated from out-ofhospital cardiac arrest. Resuscitation 1998; 38: 99-105.

8 Mair P, Mair J, Seibt I et al. - Cardiac troponin T: a new marker of myocardial tissue damage in bypass surgery. J Cardiothorac Vasc Anesth 1993; 7: 674-8.

9 Astorri E, Fiorina P, Grattagliano C et al. - Cardiac troponin $\mathrm{T}$ to evaluate myocardial protection via intermittent cold blood or continuous warm blood cardioplegia in coronary artery bypass grafting. $J$ Cardiovasc Surg (Torino) 1998; 39: 797-802.

10 Bouchard D \& Cartier R - Off-pump revascularization of multivessel coronary artery disease has a decreased myocardial infarction rate. Eur J Cardiothorac Surg 1998; 14(Suppl 1): S20-4. 